\title{
Anti-cancer effect of EGCG and its mechanisms
}

\section{Motofumi Kumazoe and Hirofumi Tachibana*}

Division of Applied Biological Chemistry, Department of Bioscience and Biotechnology, Faculty of Agriculture, Kyushu University, 6-10-1 Hakozaki, Higashi-ku, Fukuoka 812-8581, Japan

Corresponding author: Hirofumi Tachibana, PhD, Professor, 6-10-1 Hakozaki, Higashi-ku, Fukuoka 812-8581, Japan.

Submission date: October 1, 2015, Acceptance date: January 30, 2016: Publication date: February 3, 2016

\begin{abstract}
Background: Epidemiological analysis demonstrated that there are negative correlation between green tea consumption and the risk of non-Hodgkin lymphoma and prostate cancer. Recent studies show (-)-epigallocatechin-3-O-gallate (EGCG), or major green tea polyphenol, suppresses the proliferation of cancer cells and induces cell death without adversely affecting normal cells. As a result, several molecular mechanisms have been suggested to be responsible for this effect. For example, 67-kDa laminin receptor (67LR) was recently identified as the sensing molecule for EGCG. 67LR overexpresses in cancer cells and plays a crucial role in the selective toxicity of EGCG. Moreover, possible downstream mechanisms were suggested in 67LR-dependent the anti-cancer effect of EGCG. This review focused on the molecular mechanism of EGCG and developing a novel strategy to amplify its effect.
\end{abstract}

\section{Green tea shows the anti-cancer effect}

Green tea, made from the leaves of the Camellia sinensis L., is one of the most widely consumed beverages. Recently, there has been growing interest in the beneficial effects of green tea, including its anti-obesity [1], anti-inflammatory [2], cancer-preventive effects. The therapeutic activities of green tea has been demonstrated for numerous types of cancers, including oral cavity, stomach, small intestine, colon, lung, bladder, prostate and mammary glands in vivo [3].

Three case-control epidemiological studies have demonstrated that green tea consumption is associated with a reduced risk of hematopoietic cancer, leukemia and non-Hodgkin lymphoma [4]. Previous phase II clinical trial demonstrated that the oral Polyphenon $\mathrm{E}^{\mathrm{TM}}$, a $60 \%$ (-)epigallocatechin-3-gallate (EGCG)-containing botanical drug was well tolerated. Within 29 of 43 patients with chronic lymphocytic leukemia (67\%), there was evidence of a biological response with decreased lymphadenopathy and/or absolute lymphocyte count [5]. Furthermore, unlike other potential anti-cancer drug candidates, green tea extract is generally well tolerated by patients.

Several studies showed the active compounds in the green tea extract are the catechins [3, 6]. The common of which in green tea are EGCG, (-)-epigallocathechin (EGC), (-)-epicatechin- 
3-gallate (ECG), and (-)-epicatechin (EC). In those catechins, EGCG plays the crucial role in cancer-preventive and therapeutic activities of green tea extract [3].

Recent studies demonstrated that EGCG shows anti-cancer effects in several types of cancer including prostate cancer [7], melanoma [8], multiple myeloma [9, 10], acute myelogenous leukemia [11], and chronic myelogenous leukemia [12] without affecting normal cells. A significant number of different molecular mechanisms have been proposed for its anticancer effect [13-15], including the inhibition of anti-apoptosis protein, B-cell lymphoma [13], radical oxygen species (ROS) production [14], and VEGF receptor inhibition [15]. However, the concentrations of EGCG used in some of the cell culture experiments $(20-100 \mu \mathrm{M})$ are higher than the plasma observed in clinical studies [16]. Moreover, these mechanisms have not been confirmed in vivo studies. In this study, we will introduce the molecular mechanisms involved in anti-cancer effect of EGCG at physiological condition.

\section{LR is the sensing molecule of EGCG}

The 67-kD laminin receptor (67LR), also known as Ribosomal Protein SA (RPSA), is a cellsurface receptor for laminin with high affinity [17]. This receptor is peculiar, as only a fulllength gene encoding a $37-\mathrm{kDa}$ precursor protein of 295-amino acids has been isolated. 67LR is involved in adhesion in normal cells, however, several pathological studies suggested the 67LR has been shown to be overexpressed in various types of cancer including breast cancer $[17,18]$, pancreatic cancer [18], gastric cancer [18], chronic lymphocytic leukemia [19], melanoma [8], multiple myeloma [9, 10], and acute myelogenous leukemia [20].

Clinical studies have shown that upregulation of the expression level of 67LR is positively correlated with poor prognosis, histological severity of lesions, and tumor progression [17]. Cancer-upregulated 67LR plays the crucial role in abnormally upregulated expression of cyclins $\mathrm{A}$ and B and cyclin-dependent kinases 1 and 2 [21]. Moreover, 67LR is involved in adhesionmediated drug resistance [22].

All-trans-retinoic acid increased the binding of EGCG to the cell surface of cancer cells when the binding was monitored on the based on surface plasmon resonance assay. In order to identify candidates for the sensing molecule of EGCG, we used a subtraction cloning technique based on cDNA pool constructed from cells treated or untreated with all-trans-retinoic acid [23]. Based on analysis of the subtraction cloning, 67LR was identified as the sensing molecule of EGCG [23, 24].

The binding of EGCG on 67LR was assessed in experiments using surface plasmon resonance, and Kd was also assessed. Our data suggested Kd value for the binding of EGCG to the $67 \mathrm{LR}$ [23] is $39.9 \mathrm{nM}$. Moreover, this anti-cancer effect of EGCG was significantly neutralized upon treatment with anti-67 LR antibody before the addition of EGCG [10, 18, 25].

In the animal model, we previously reported EGCG did not show anti-cancer effects in 67LR-knockdowned B16 mouse melanoma xenograft model, while it significantly reduced tumor growth in scramble vector transfected B16 mouse melanoma xenograft [26]. When taken together, 67LR plays a crucial role in the anti-cancer effects of EGCG in vivo and its selective toxicity.

Several in vitro and in vivo experiments suggested EGCG induced cell growth inhibition and cell death in many types of cancer without affecting normal cells. Interestingly, Shammas et. al. 
reported, 67LR were highly overexpressed in multiple myeloma cell lines and primary multiple myeloma cells compared with its normal counterparts [9]. Exposure of EGCG led to apoptotic cell death in 67LR-overexpressed multiple myeloma cells. Their results showed higher susceptibility of multiple myeloma cells to EGCG, which may provide a higher therapeutic index.

The same results were obtained from several types of cancer, including, acute myelogenous leukemia [11, 20], melanoma [8, 27], and chronic lymphocytic leukemia [19], 67LR, sensing molecule of EGCG are strongly upregulated in cancer cells compared with its normal counterpart. Our ex vivo study based on chronic lymphocytic leukemia cells from 13 patients demonstrated there are positive correlation between the expression level of 67LR and EGCG susceptibility [19].

Furthermore, we previously reported how there is a sensing motif of 67LR to EGCG. Only a 10-aminoacids, corresponding to 161 to 170 , IPCNNKGAHS, is involved in 67LR-mediated cellular response to EGCG stimulation [28]. This is the first evidence indicating the existence of a potential EGCG sensing motif on 67LR.

\section{Downstream mechanisms after EGCG bind to 67LR}

Because Gloe $\mathrm{T}$ et. al. reported that $67 \mathrm{LR}$ plays an important role in shear stress-induced endothelial nitric oxide synthase (eNOS)-dependent signalling [29], we assessed the role of nitric oxide (NO) in 67LR-dependent cell death [18]. EGCG induced NO production in primary multiple myeloma cells and multiple myeloma cell lines without affecting normal peripheral blood mononuclear cells. Moreover, we demonstrated how EGCG induced eNOS phosphorylation at Ser1177 plays a crucial role in eNOS activity [11, 18]. Importantly, EGCGinduced eNOS phosphorylation at Ser1177 was canceled by pretreatment of multiple myeloma cells with an anti-67LR monoclonal antibody [18].

cGMP is known as a second messenger, or the crucial mediator in NO-induced signaling [30]. We revealed that EGCG increased cGMP in multiple myeloma cells [18] and acute myeloid leukemia [11], but had no effect on normal peripheral blood mononuclear cells. NO upregulates the intercellular cGMP level by activating soluble guanylate cyclase (sGC). Significantly, the sGC inhibitor NS-2028 prevented both EGCG-induced cGMP upregulation and cell death [18]. Therefore, eNOS/NO/sGC/cGMP signal pathway is indispensable for anti-cancer effect of EGCG.

Acid sphingomyelinase (ASM) generates ceramide as a lipid second messenger, or a potent inducer of increasing membrane fluidity and apoptosis [31]. Because EGCG induced lipid-raft clustering via 67LR, we assessed the effect of EGCG on ASM. Our results suggested EGCG induce ASM activation in multiple myeloma [10], acute myelogenous leukemia [11], and chronic myelogenous leukemia [32] without affecting ASM activity in normal peripheral blood mononuclear cells [10]. We confirmed ASM inhibitor, or desipramine, strongly cancelled EGCG-induced cell death in those types of hematopoietic cancer [10, 11, 32].

Protein Kinase $\mathrm{C}$ delta (PKCS) is one of the novel PKCs activated by diacylglycerol or 12Otetradecanoylphorbol 13-acetate [33]. PKC $\delta$ plays the crucial role in activation of ASM. The selective phosphorylation of PKC $\delta$ at Ser664, but Tyr155 was not observed in EGCG-treated multiple myeloma cells [10]. The same results were obtained from chronic lymphoid leukemia 
cells [19]. As a result, we clarified that eNOS/NO/sGC/cGMP/PKCS/ASM axis induced by EGCG bound to its sensing molecule. 67LR is an indispensable signalling for this cell death pathway (Figure 1A).

To reveal the effector molecule in growth inhibition effect of EGCG, we used genetic suppressor element (GSE) method; cDNA fragments acting as dominant inhibitors of protein function or RNAi [34]. GSEs act as selectable markers through the change in phenotype based on repression of the gene, thus allowing identification of genes, a process which plays a crucial role in drug susceptibility [26, 8]. To screen genes involved in EGCG sensitivity, we selected GSE-transfected clones conferring resistance to EGCG. Our analysis demonstrated how GSE encoded protein phosphatase 2A (PP2A), which protected melanoma from anti-cancer effect of EGCG [8]. Tumor volume increase was completely suppressed in EGCG-treated mice implanted with control shRNA B16 cells, whereas EGCG did not exhibit anti-cancer effect in mice implanted with PP2A shRNA B16 cells [8]. We demonstrated that tumor-overexpressed 67LR activates PP2A through adenylate cyclase/cAMP pathway [8] (Figure 1A).

Surprisingly, Santilli et. al. demonstrated how Polyphenon E suppressed the development and motility of myeloid-derived suppressor cells (MDSCs) and enhance differentiation to more neutrophilic forms through the 67LR signaling and the detail further investigation is strongly demanded [35].

\section{Searching negative regulators could be attractive to amplify anti-cancer effect of EGCG "The true way to Paradise - Learn the way to Hell in order to flee from it"}

Our studies showed high EGCG concentrations $(20 \mu \mathrm{M})$ were necesscary to elicit cell death [18], even though the dissociation constant of EGCG to 67LR is $0.04 \mu \mathrm{M}$ [23]. EGCG at physiologically achievable levels $(5 \mu \mathrm{M})$ could induce NO production, but could not increase intracellular cGMP level to induce multiple myeloma cell death [18]. Those findings indicated that induction of cGMP may be a "choke point" of the EGCG-elicited cell death pathway (Figure 1A).

Phosphodiesterases (PDEs), well-known negative regulators of cGMP degrades the 3',5'phosphodiester bond of cGMP. This is because the characteristics of each isoforms of PDEs are involved in specific signaling pathways, PDEs could be ideal drug targets, due to selective inhibition of PDE isoforms [36].

Our results suggested a PDE5-selective inhibitor, vardenafil used for treating erectile dysfunction, potentiated the anticancer effect of the EGCG in multiple myeloma [18], acute myelogenous leukemia [11], and chronic lymphocytic leukemia [19]. Moreover, PDE5 expression was highly upregulated in multiple myeloma, acute myelogenous leukemia, chronic lymphocytic leukemia, gastric cancer, stomach cancer, and breast cancer [18]. EGCG and vardenafil in combination resulted in greater inhibition of the cancer cell growth of multiple myeloma cells, with an $\mathrm{IC}_{50}$ of $1.4 \mu \mathrm{M}$ compared with $23.2 \mu \mathrm{M}$ for EGCG alone [18].

Suvar3-9, enhancer-of-zeste, trithorax (SET) is a protein, isolated from a chromosomal rearrangement at 9q34 in a patient with leukemia [37] plays the crucial role in inhibition of PP2A.

We demonstrated how SET is highly expressed in melanoma patient tissue compared with normal cells [8]. Indeed, the silencing of SET amplified EGCG-induced PP2A activity and 
significantly inhibited B16 cell proliferation both in vitro and in vivo [8]. These findings suggested inhibition of SET might be a potential strategy to amplify the effect of EGCG.

Recently, we also demonstrated how negative regulator of ceramide, sphingosine kinase 1 (SphK1) is over-expressed in multiple myeloma cells compared with its normal counterpart [38]. We demonstrate that over-expression of SphK1 attenuates EGCG-induced cell death. Moreover, safingol, SphK1 inhibitor synergically potentiated anti-cancer effect of EGCG both in vitro and in vivo model [38]. Taken together, combined regimen for striking crucial negative regulator could be a novel tactics for overcoming EGCG resistance (Figure 1B).

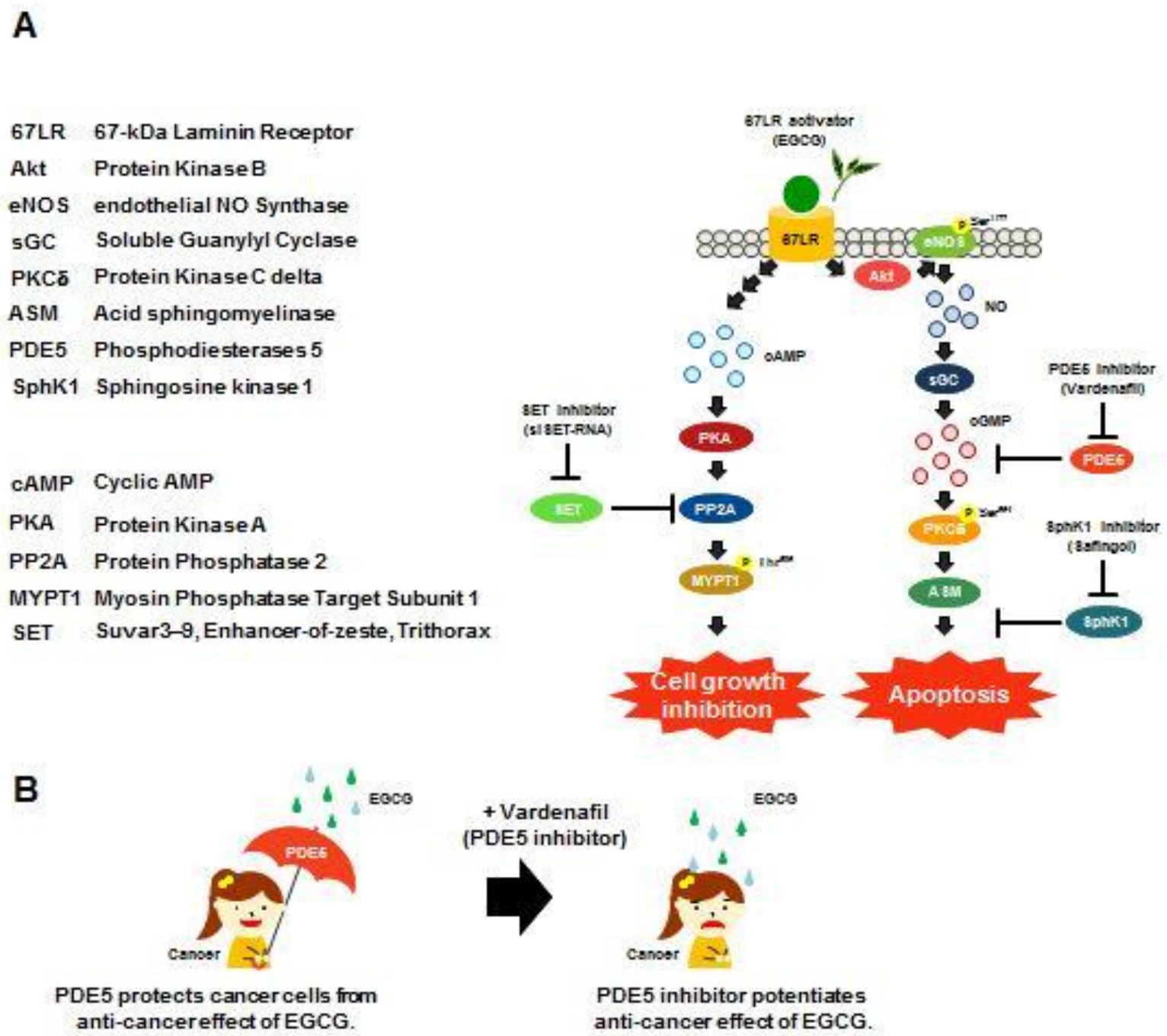

Figure 1. Searching for negative regulators could be attractive to amplify anti-cancer effect of EGCG.

(A) 67LR-dependent signaling pathway

(B) PDE5 protects cancer cells from EGCG-induced cell death 


\section{CONCLUSIONS}

We identified 67LR as the sensing molecule of EGCG, and also revealed the downstream mechanism of EGCG-induced cell death and growth inhibition. Our studies demonstrated that 67LR acts as the cancer-overexpressed death receptor which induces the apoptotic signaling pathway. Our finding revealed that 67LR could be an attractive target for cancer chemotherapy and provide a rationale for the clinical value of EGCG as a 67LR-targeting drug. Based on the molecule mechanisms of EGCG-induced anti-cancer effect, we revealed how PDE5, SET and SphK1 act as resistant factors against EGCG, and also provided a novel strategy to amplify its anti-cancer effect.

\section{List of abbreviations:}

67-kDa laminin receptor, 67LR; acid sphingomyelinase, ASM; (-)-epicatechin-3-gallate, ECG; (-)-epicatechin, EC; (-)-epigallocathechin, EGC; (-)-epigallocatechin-3-O-gallate, EGCG; endothelial nitric oxide synthase, eNOS; genetic suppressor element, GSE; myeloid-derived suppressor cells, MDSCs; nitric oxide, NO; radical oxygen species, ROS; Ribosomal Protein SA, RPSA; Protein Kinase C delta, PKC $\delta$; Phosphodiesterase, PDE; protein phosphatase 2A, PP2A; Suvar3-9, enhancer-of-zeste, trithorax, SET; soluble guanylate cyclase, sGC; sphingosine kinase $1, \mathrm{SphK} 1$

Competing Interests: The authors have no financial interests or any other conflicts of interest to disclose.

Acknowledgments: This work was supported in part by JSPS KAKENHI grant 22228002 and $15 \mathrm{H} 02448$ to H. Tachibana and M. Kumazoe 15K18821. The pictures were drawn by Kanako Takamatsu. The authors declare no competing financial interests.

Authors' Contributions: All authors contributed to this review.

\section{REFERENCES:}

1. Suzuki, T., Kumazoe, M., Kim, Y., Yamashita, S., Nakahara, K., Tsukamoto, S., Sasaki, M., Hagihara, T., Tsurudome, Y., Huang, Y., Maeda-Yamamoto, M., Shinoda, Y., Yamaguchi, W., Yamada, K. and Tachibana, H. Green tea extract containing a highly absorbent catechin prevents diet-induced lipid metabolism disorder. Sci. Rep. 2013, 3, 2749

2. Byun, E. H., Omura, T., Yamada, K. and Tachibana, H. Green tea polyphenol epigallocatechin-3-gallate inhibits TLR2 signaling induced by peptidoglycan through the polyphenol sensing molecule 67-kDa laminin receptor. FEBS Lett. 2011, 585 (5), 814820.

3. Yang, C. S. and Wang, H. Cancer therapy combination: green tea and a phosphodiesterase 5 inhibitor? J. Clin. Invest. 2013, 123:1-3.

4. Naganuma, T., Kuriyama, S., Kakizaki, M., Sone, T., Nakaya, N., Ohmori-Matsuda, K., Hozawa, A. Nishino, Y. and Tsuji, I. Green tea consumption and hematologic 
malignancies in Japan: the Ohsaki study. Am. J. Epidemiol. 2009, 170:730-738.

5. Shanafelt, T. D., Call, T. G., Zent, C. S., Leis, J. F., LaPlant, B., Bowen, D. A., Roos, M., Laumann, K., Ghosh, A. K., Lesnick, C., Lee, M. J., Yang, C. S., Jelinek, D. F., Erlichman, C. and Kay, N. E. Phase 2 trial of daily, oral Polyphenon E in patients with asymptomatic, Rai stage 0 to II chronic lymphocytic leukemia. Cancer 2013, 119:363370.

6. Kumazoe, M., Fujimura, Y., Hidaka, S., Kim, Y., Murayama, K., Takai, M., Huang, Y., Yamashita, S., Murata, M., Miura, D., Wariishi, H., Maeda-Yamamoto, M., Tachibana, H. Metabolic Profiling-based Data-mining for an Effective Chemical Combination to Induce Apoptosis of Cancer Cells. Sci. Rep. 2015, 5:9474.

7. Kim, S. J., Amankwah, E., Connors, S., Park, H. Y., Rincon, M., Cornnell, H., Chornokur, G., Hashim, A. I., Choi, J., Tsai, Y. Y., Engelman, R. W., Kumar, N. and Park, J. Y. Safety and chemopreventive effect of Polyphenon E in preventing early and metastatic progression of prostate cancer in TRAMP mice. Cancer Prev. Res. 2014, 7:435-444.

8. Tsukamoto, S., Huang, Y., Umeda, D., Yamada, S., Yamashita, S., Kumazoe, M., Kim, Y., Murata, M., Yamada, K. and Tachibana, H. 67-kDa Laminin Receptor-dependent Protein Phosphatase 2A (PP2A) Activation Elicits Melanoma-specific Antitumor Activity Overcoming Drug Resistance. J. Biol. Chem. 2014, 289: 32671-32681.

9. Shammas, M. A., Neri, P., Koley, H., Batchu, R. B., Bertheau, R. C., Munshi, V., Prabhala, R., Fulciniti, M., Tai, Y. T., Treon, S. P., Goyal, R. K., Anderson, K. C. and Munshi, N. C. Specific killing of multiple myeloma cells by (-)-epigallocatechin-3gallate extracted from green tea: biologic activity and therapeutic implications. Blood 2006, 108:2804-2810.

10. Tsukamoto, S., Hirotsu, K., Kumazoe, M., Goto, Y., Sugihara, K., Suda, T., Tsurudome, Y., Suzuki, T., Yamashita, S., Kim, Y., Huang, Y., Yamada, K. and Tachibana, H. Green tea polyphenol EGCG induces lipid-raft clustering and apoptotic cell death by activating protein kinase $\mathrm{C}$ delta and acid sphingomyelinase through a $67 \mathrm{kDa}$ laminin receptor in multiple myeloma cells. Biochem. J. 2012, 443 (2), 525-534.

11. Kumazoe, M., Kim, Y., Bae, J., Takai, M., Murata, M., Suemasu, Y., Sugihara, K., Yamashita, S., Tsukamoto, S., Huang, Y., Nakahara, K., Yamada, K. and Tachibana, H. Phosphodiesterase 5 inhibitor acts as a potent agent sensitizing acute myeloid leukemia cells to 67-kDa laminin receptor-dependent apoptosis. FEBS Lett. 2013, 587, 3052-3057.

12. Huang, Y., Kumazoe, M., Bae, J., Yamada, S., Takai, M., Hidaka, S., Yamashita, S., Kim, Y., Won, Y., Murata, M., Tsukamoto, S. and Tachibana, H. Green tea polyphenol epigallocatechin- $O$-gallate induces cell death by acid sphingomyelinase activation in chronic myeloid leukemia cells. Oncol. Rep. 2015, 34 (3), 1162-1168.

13. Leone, M., Zhai, D., Sareth, S., Kitada, S., Reed, J. C. and Pellecchia, M. Cancer prevention by tea polyphenols is linked to their direct inhibition of antiapoptotic Bcl-2family proteins. Cancer Res. 2003, 63 (23), 8118-8121.

14. Nakazato, T., Ito, K., Ikeda, Y. and Kizaki, M. Green tea component, catechin, induces apoptosis of human malignant B cells via production of reactive oxygen species. Clin. Cancer Res. 2005, 11 (16), 6040-6049. 
15. Lee, Y. K., Bone, N. D., Strege, A. K., Shanafelt, T. D., Jelinek, D. F. and Kay, N. E. VEGF receptor phosphorylation status and apoptosis is modulated by a green tea component, epigallocatechin-3-gallate (EGCG), in B-cell chronic lymphocytic leukemia. Blood 2004, 104 (3), 788-794.

16. Yang, C. S., Wang, X., Lu, G. and Picinich, S. C. Cancer prevention by tea: animal studies, molecular mechanisms and human relevance. Nature Reviews Cancer 2009, 9 (6), 429-439.

17. Viacava, P., Naccarato, A. G., Collecchi, P., Menard, S., Castronovo, V. and Bevilacqua, $\mathrm{G}$. The spectrum of $67-\mathrm{kD}$ laminin receptor expression in breast carcinoma progression. J. Pathol. 1997, 182 (1), 36-44.

18. Kumazoe, M., Sugihara, K., Tsukamoto, S., Huang, Y., Tsurudome, Y., Suzuki, T., Suemasu, Y., Ueda, N., Yamashita, S., Kim, Y., Yamada, K. and Tachibana, H. 67-kDa laminin receptor increases cGMP to induce cancer-selective apoptosis. J. Clin. Invest. 2013, 123 (2), 787-799.

19. Kumazoe, M., Tsukamoto, S., Lesnick, C., Kay, N. E., Yamada, K., Shanafelt, T. D. and Tachibana, H. Vardenafil, a clinically available phosphodiesterase inhibitor, potentiates the killing effect of EGCG on CLL cells. Br. J. Haematol. 2015, 168, 610-613.

20. Britschgi, A., Simon, H. U., Tobler, A., Fey, M. F. and Tschan, M. P., Epigallocatechin-3gallate induces cell death in acute myeloid leukaemia cells and supports all-trans retinoic acid-induced neutrophil differentiation via death-associated protein kinase 2. Br. J. Haematol. 2010, 149 (1), 55-64.

21. Scheiman, J., Tseng, J. C., Zheng, Y. and Meruelo, D. Multiple Functions of the 37/67kd Laminin Receptor Make It a Suitable Target for Novel Cancer Gene Therapy. Molecular Therapy 2009, 18 (1), 63-74.

22. Liu, L., Sun, L., Zhang, H., Li, Z., Ning, X., Shi, Y., Guo, C., Han, S., Wu, K. and Fan, D. Hypoxia-mediated up-regulation of MGr1-Ag/37LRP in gastric cancers occurs via hypoxia-inducible-factor 1-dependent mechanism and contributes to drug resistance. International journal of cancer. J. Int. Cancer 2009, 124 (7), 1707-1715.

23. Tachibana, H., Koga, K., Fujimura, Y. and Yamada, K. A receptor for green tea polyphenol EGCG. Nat. Struct. Mol. Biol. 2004, 11 (4), 380-381.

24. Kumazoe, M. and Tachibana, H. 67-kDa laminin receptor and cGMP induced cancerselective apoptosis. BMC Pharmacol. Toxicol. 2013, 14, O33.

25. Lee, J. H., Kishikawa, M., Kumazoe, M., Yamada, K. and Tachibana, H. Vitamin A enhances antitumor effect of a green tea polyphenol on melanoma by upregulating the polyphenol sensing molecule 67-kDa laminin receptor. PLoS One 2010, 5 (6), e11051.

26. Umeda, D., Yano, S., Yamada, K. and Tachibana, H. Green tea polyphenol epigallocatechin-3-gallate signaling pathway through $67-\mathrm{kDa}$ laminin receptor. J. Biol. Chem. 2008, 283 (6), 3050- 3058.

27. Tsukamoto, S., Yamashita, S., Kim, Y. H., Kumazoe, M., Huang, Y., Yamada, K. and Tachibana, H., Oxygen partial pressure modulates 67-kDa laminin receptor expression, leading to altered activity of the green tea polyphenol, EGCG. FEBS Lett. 2012, 586 (19), 3441-3447.

28. Fujimura, Y., Sumida, M., Sugihara, K., Tsukamoto, S., Yamada, K. and Tachibana, H. 
Green tea polyphenol EGCG sensing motif on the 67-kDa laminin receptor. PLOS ONE 2012, 7 (5), e37942.

29. Gloe, T., Riedmayr, S., Sohn, H. Y. and Pohl, U. The 67-kDa laminin-binding protein is involved in shear stress-dependent endothelial nitric-oxide synthase expression. J. Biol. Chem. 1999, 274 (23), 15996-6002.

30. Arnold, W. P., Mittal, C. K., Katsuki, S. and Murad, F. Nitric oxide activates guanylate cyclase and increases guanosine 3':5'-cyclic monophosphate levels in various tissue preparations. Proc. Natl. Acad. Sci. USA 1977, 74 (8), 3203-3207

31. Lacour, S., Hammann, A., Grazide, S., Lagadic-Gossmann, D., Athias, A., Sergent, O., Laurent, G., Gambert, P., Solary, E. and Dimanche-Boitrel, M. T. Cisplatin-induced CD95 redistribution into membrane lipid rafts of HT29 human colon cancer cells. Cancer Res. 2004, 64, 3593-3598.

32. Huang, Y., Kumazoe, M., Bae, J., Yamada, S., Takai, M., Hidaka, S., Yamashita, S., Kim, Y., Won, Y., Murata, M., Tsukamoto, S. and Tachibana, H. Green tea polyphenol epigallocatechin-O-gallate induces cell death by acid sphingomyelinase activation in chronic myeloid leukemia cells. Oncol. Rep. 2015, 34 (3), 1162-1168.

33. Efimova, T. and Eckert, R.L. Regulation of human involucrin promoter activity by novel protein kinase C isoforms. J. Biol. Chem. 2000, 275(3), 1601-1607.

34. Roninson, I. B., Gudkov, A. V., Holzmayer, T. A., Kirschling, D. J., Kazarov, A. R., Zelnick, C. R., Mazo, I. A., Axenovich, S. and Thimmapaya, R. Genetic Suppressor Elements: New Tools for Molecular Oncology. Cancer Res. 1995, 55, 4023-4028

35. Santilli, G., Piotrowska, I., Cantilena, S., Chayka, O., D'Alicarnasso, M., Morgenstern, D. A., Himoudi, N., Pearson, K., Anderson, J., Thrasher, A. J. and Sala, A. Polyphenol E enhances the antitumor immune response in neuroblastoma by inactivating myeloid suppressor cells. Clin. Cancer Res. 2013, 19 (5), 1116-1125.

36. Lugnier, C. Cyclic nucleotide phosphodiesterase (PDE) superfamily: a new target for the development of specific therapeutic agents. Pharmacol. Ther. 2006, 109 (3), 366-98.

37. Li, M., Makkinje, A. and Damuni, Z. The myeloid leukemia-associated protein SET is a potent inhibitor of protein phosphatase 2A. J. Biol. Chem. 1996, 271 11059-11062

38. Tsukamoto, S., Huang, Y., Kumazoe, M., Lesnick, C., Yamada, S., Ueda, N., Suzuki, T., Yamashita, S., Kim, Y. H., Fujimura, Y., Miura, D., Kay, N. E., Shanafelt, T. D. and Tachibana, H. Sphingosine kinase-1 protects multiple myeloma from apoptosis driven by cancer specific inhibition of RTKs. Mol. Cancer. Ther. 2015, 14 2303-2312 i 